EPJ Web of Conferences 16, 02007 (2011)

DOI: $10.1051 /$ epjconf/20111602007

(C) Owned by the authors, published by EDP Sciences, 2011

\title{
Improving the radial velocity precision of NIRSPEC data
}

\author{
F. Rodler ${ }^{1, a}$, E.L. Martín², M.R. Zapatero Osorio², R. Deshpande ${ }^{3}$ \\ and C. del Burgo ${ }^{4,5}$ \\ ${ }^{1}$ Instituto de Astrofísica de Canarias, C/Via Láctea s/n, 38205 La Laguna, Spain \\ 2 Centro de Astrobiología (CAB-CSIC), Ctra. Ajalvir km. 4, 28850 Torrejón de Ardoz, Madrid, \\ Spain \\ ${ }^{3}$ University of Central Florida, Physics Department, PO Box 162385, Orlando, FL 32816, USA \\ ${ }^{4}$ School of Cosmic Physics, Dublin Institute of Advance Studies, Ireland \\ ${ }^{5}$ UNINOVA-CA3, Campus da Caparica, Quinta da Torre, Monte de Caparica, \\ 2825-149 Caparica, Portugal
}

\begin{abstract}
We present an approach to improve the radial velocity precision of NIRSPEC data (spectral resolution $R=\lambda / \Delta \lambda \approx 22,000$ ) by using a self-calibration approach. We demonstrate that the accuracy can be significantly improved by deconvolving the spectra and using the telluric lines as a long-term stable wavelength reference.
\end{abstract}

\section{INTRODUCTION}

Up to now, more than 400 planets beyond our Solar System have been detected. One of the primary goals is to detect (and study) Earth-like planets. Low mass stars such as M-dwarfs are suitable targets to search for low-mass planets by adopting the radial velocity (RV) technique (e.g. Mayor \& Queloz 1995 [1], Marcy 1998 [2]). These stars radiate most of their energy at near-infrared wavelength (NIR); therefore observations in this wavelength regime are carried out (Martín et al. 2005 [3], 2006 [4]). Here, we report a strategy to improve the RV-precision of measurements of $16 \mathrm{M}$-dwarfs (spectral types M5V-M9V), which were part of a large observation programme (Deshpande et al. 2008 [5]). Observations were carried out in the J-band using the cross-dispersed spectrograph NIRSPEC (McLean et al. 1998 [6]), mounted on the Keck II telescope on Mauna Kea, Hawaii, with a spectral resolution of $R=\lambda / \Delta \lambda \approx 22,000$.

\section{STRATEGY}

The main idea is to use the telluric lines which are present in most of the spectral orders as natural gas cell providing a stable reference, and to self-calibrate the data (e.g. Endl et al. 2000 [7]). In addition to that, the spectra are corrected for variations of the instrumental profile (IP), which mainly come from changes in the spectrograph.

Step1: In the first step, we redo the wavelength calibration, but using the telluric lines instead of the arc-lamp lines (Figure 1, right hand panel). We have used the Earth transmission spectrum above Mauna Kea, generated with the HITRAN ${ }^{1}$ code. We note that we use vacuum wavelengths. Due to wind and

\footnotetext{
ae-mail: frodler@iac.es

${ }^{1}$ HITRAN data base: http: //www. cfa.harvard.edu/hitran/
}

This is an Open Access article distributed under the terms of the Creative Commons Attribution-Noncommercial License 3.0, which permits unrestricted use, distribution, and reproduction in any noncommercial medium, provided the original work is properly cited. 

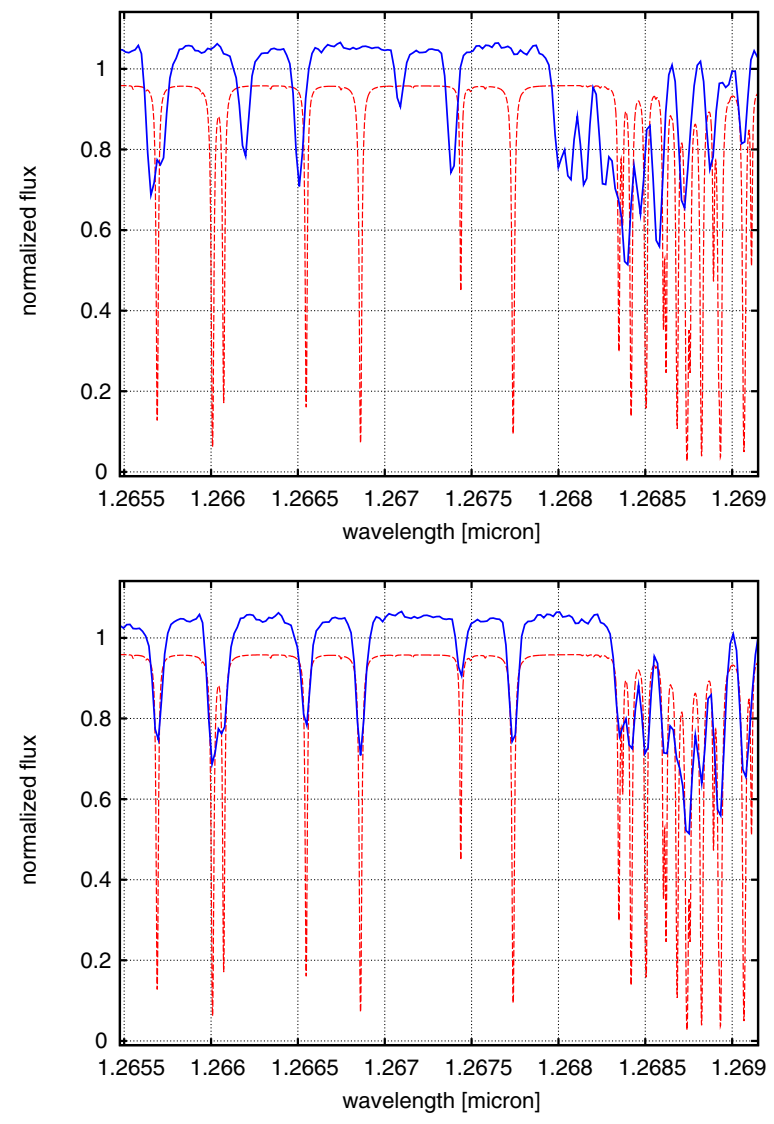

Figure 1. Left hand panel. The solid line depicts a part of the observed telluric lines (by taking measurments of a fast-rotating A-type star, which shows no intrinsic features in the NIR), while the dashed line shows the calculated telluric HITRAN spectrum (vacuum wavelengths). The observed spectrum was wavelength-calibrated by using the arc-lamp of NIRSPEC. Right hand panel. The same observed spectrum after having applied the wavelength calibration using the telluric lines (vacuum wavelength values).

other atmospheric effects, the maximum wavelength precision which can be achieved using these lines is approx. $10 \mathrm{~m} / \mathrm{s}$ (e.g. Seifahrt \& Käufl 2008 [8]) - i.e. for our purposes more than sufficient.

Step 2: This wavelength-calibrated spectrum of the telluric standard (i.e. a fast-rotating A- type star, which is featureless in the J-band) is deconvolved by using the HITRAN-spectrum, adopting LeastSquares deconvolution. This gives us the IP (Figure 2, left). The IP is then improved by a multi-gauss approach (cf. Endl et al. 2000 [7], Valenti et al. 1995 [9]).

Step 3: Knowing the IP for each spectral order, all the target + telluric standard spectra are deconvolved by using the Maximum Entropy Method; in this step we remove the IP from the spectra, and consequently, sharpen the spectra.

Step 4: Now we calibrate the deconvolved spectrum of the telluric standard using the telluric lines (Figure 2, right). This refined wavelength calibration is then applied to the deconvolved science + tellurics spectrum. We note that the telluric lines are not removed from the target spectra.

\section{DETERMINATION OF THE RV-SHIFTS}

Finally, the RVs of the science targets are determined. To this end, we construct a model describing the M-dwarf plus the telluric lines consisting of the HITRAN spectrum and an appropriate stellar 

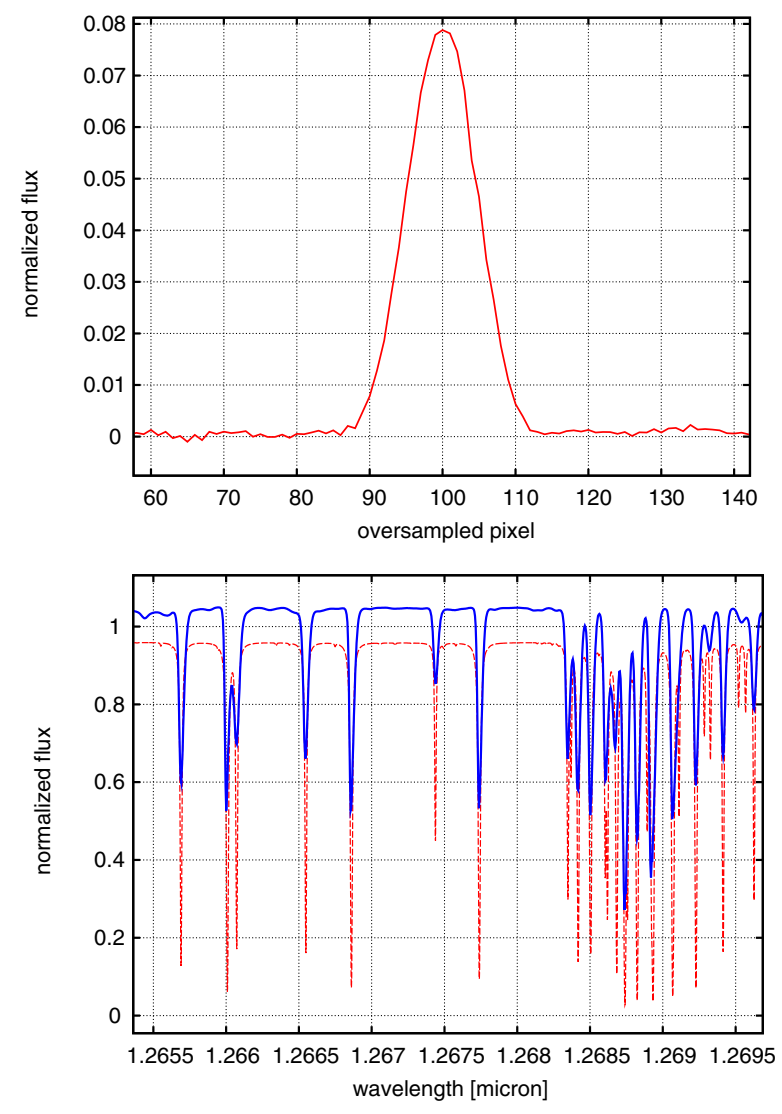

Figure 2. Left hand panel. Instrumental profile, which was determined by a Least-Squares deconvolution of the observed spectrum of the telluric standard (A-type star) with a model spectrum of the telluric lines, calculated by using the HITRAN code. Right hand panel. The observed spectrum of the telluric standard after having removed the instrumental profile (solid line). This spectrum was then wavelength calibrated by using the sharpened telluric lines (dashed).

atmosphere synthetic model for the M-dwarf (see del Burgo et al., these proceedings; del Burgo et al. 2009 [10]).

We subdivide each spectral order containing a large number of telluric lines into 4 chunks. For each chunk, we construct the aforementioned model, which is into characterized by five free parameters: first and second order terms of diplacement of the HITRAN spectrum with respect to the observations, and a scaling factor; RV-shift of the stellar model and scaling factor with respect to the observation. The best fit model is then evaluated by $\chi^{2}$-minimization, and the RV-shift of the star finally determined per chunk. Once having all RVs of all chunks combined, we achieve an RV-precision of 120-150 m/s for M-dwarfs, which constitutes a huge improvement of the RV-precision of NIRSPEC. Previous analysis of the same data set resulted in a RV-precision around $300 \mathrm{~m} / \mathrm{s}$ (Zapatero Osorio et al. 2009 [11]).

\section{CONCLUSIONS AND OUTLOOK}

We have demonstrated that the accuracy of the wavelength calibration of NIRSPEC data with spectral resolution around 20,000 can be significantly improved by correcting the spectra of the IP and using the telluric lines as reference. By adopting the self-calibration technique, we lower the RV-uncertainties 
to $120-150 \mathrm{~m} / \mathrm{s}$ per measurement (i.e. all spectral orders used combined), which is a significant improvement regarding to previous analyses.

This work has been supported by the Spanish Ministerio de Eduación y Ciencia through grant AYA2007-67458.

\section{References}

[1] Mayor M. \& Queloz D. 1995, Nature, 378, 355

[2] Marcy G. W. 1998, Nature, 391, 127

[3] Martín E. L., Guenther E., et al., 2005, AN, 326, 1015

[4] Martín E. L., Guenther E., et al., 2006, ApJ, 644, L75

[5] Deshpande R., Martín E. L., Montgomery M. M., et al., 2008, AAS meeting abstact, 221, 159.03

[6] McLean I. et al. 1998, SPIE, 3354, 566

[7] Endl M., Kürster M., Els, S., 2000, A\&A, 362, 583

[8] Seifahrt A. \& Käufl H. U. 2008, A\&A, 491, 929

[9] Valenti J. A., Butler, R. P., Marcy J. W., 1995, PASP, 107, 966V

[10] del Burgo C., Martín E. L., et al., A\&A, 501, 1059

[11] Zapatero Osorio M.R., et al., 2009, A\&A, 505, 5 